\title{
IRREDUCIBLE JULIA SETS OF RATIONAL FUNCTIONS
}

\author{
CLINTON P. CURRY
}

\begin{abstract}
We prove that a polynomial Julia set which is a finitely irreducible continuum is either an arc or an indecomposable continuum. For the more general case of rational functions, we give a topological model for the dynamics when the Julia set is an irreducible continuum and all indecomposable subcontinua have empty interior.
\end{abstract}

\section{INTRODUCTION}

Let $\widehat{\mathbb{C}}$ denote the Riemann sphere, and let $R: \widehat{\mathbb{C}} \rightarrow \widehat{\mathbb{C}}$ be a rational function. The Fatou set $F(R)$ is the domain of normality of the iterates $\left\{R^{i} \mid i \in \mathbb{N}\right\}$. The Julia set $J(R)$ is $\widehat{\mathbb{C}} \backslash F(R)$, which is generally regarded as the subset of $\widehat{\mathbb{C}}$ where $R$ is chaotic. If the degree of $R$ is at least two, then $J(R)$ is a non-empty, compact, perfect subset of $\widehat{\mathbb{C}}$. A continuum is a non-empty, compact, and connected metric space. A connected Julia set of a rational function is therefore a subcontinuum of $\widehat{\mathbb{C}}$.

This paper is motivated by recent work that addressed a conjecture of P. M. Makienko. The exact statement is not important to the subject of this paper; interested parties are referred to CMMR for more information. What is important is the conclusion: We proved that, if $R$ is a rational function that is a counterexample to Makienko's conjecture, its Julia set $J(R)$ is a finitely irreducible continuum, and hence an indecomposable continuum.

Definition 1 (Irreducible). A continuum $X$ is irreducible about a set $A \subset X$ if no proper subcontinuum of $X$ contains $A$. If $X$ is irreducible about a finite subset, it is called finitely irreducible. If $X$ is irreducible about a two-point subset, it is simply called irreducible.

Remark 2. The unit interval $[0,1]$ is an irreducible continuum, since no proper subcontinuum contains both of its endpoints. For the same reason, a finite tree is a finitely irreducible continuum. Conversely, any locally connected finitely irreducible continuum is a finite tree, since locally connected continua are also arcwise connected. The arc is the only locally connected irreducible continuum.

It is well-known that there are Julia sets of rational functions which are irreducible continua. For example, the Julia set of the polynomial $z \mapsto z^{2}-2$ is the interval $[-2,2]$. Though it is not known if every Julia set which is an irreducible continuum is an arc, there are far more complicated examples of irreducible continua.

Date: November 12, 2018.

2000 Mathematics Subject Classification. Primary 37F10, Secondary 54F15.

The author was supported in part by NSF-DMS-0353825. 

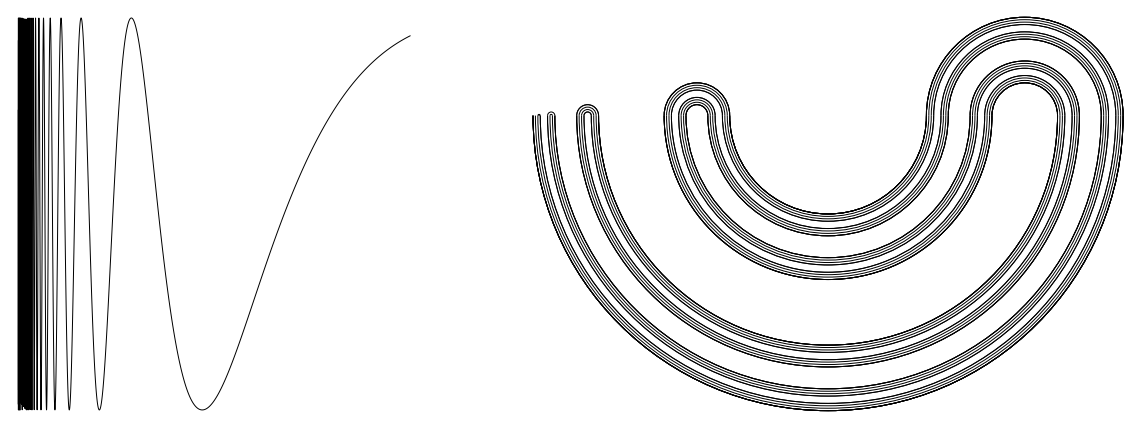

Figure 1. On the left is the $\sin \frac{1}{x}$ continuum, the simplest nonlocally connected irreducible continuum. On the right is the Knaster continuum, the simplest indecomposable continuum.

Definition 3 (Indecomposable). A continuum $X$ is decomposable if there are proper subcontinua $A$ and $B$ such that $A \cup B=X$. Otherwise, $X$ is indecomposable.

Remark 4. Indecomposable continua are also irreducible continua, even strongly so. If $X$ is an indecomposable continuum, then for a residual set of pairs $(x, y) \in$ $X \times X, X$ is irreducible between $x$ and $y$.

The arc and indecomposable continua represent the extremes of topological complexity for irreducible continua. The goal of this paper is to prove that these two cases are representative for rational Julia sets which are finitely irreducible continua. We prove in Section 4 that if $P$ is a polynomial such that $J(P)$ is an irreducible continuum, then $J(P)$ is either an arc or an indecomposable continuum. We conjecture the same result holds for rational functions, but we only prove a weaker structure theorem for rational functions using the same tools (see Section 3 ).

The argument naturally divides into two cases. The first case is when the Julia set in question is an irreducible continuum which has an indecomposable subcontinuum with non-empty interior relative to the Julia set. Not much is said about the Julia set in this case, either topologically or dynamically. The other case is that the Julia set contains no indecomposable subcontinuum with interior, which is the case that most of the statements in this paper address. To avoid awkward repetition, we introduce the following terminology.

Terminology. A continuum which is finitely irreducible such that no indecomposable subcontinuum has interior will be called a finished continuum.

Acknowledgements. I would like to express my appreciation for what Professor Devaney has done for the field and for the reserachers who work in it. I would also like to thank my doctoral advisors, Dr. Alexander Blokh and Dr. John C. Mayer, for helpful conversations on this and other topics.

\section{Aposyndesis And Vought's Decomposition}

In this section, we review the notion of aposyndesis due to Jones Jon41, Jon52. and an associated decomposition defined by Vought Vou74. The goal is to define a monotone map $m: J(R) \rightarrow Y$, where $Y$ is a topologically simpler space. In our 


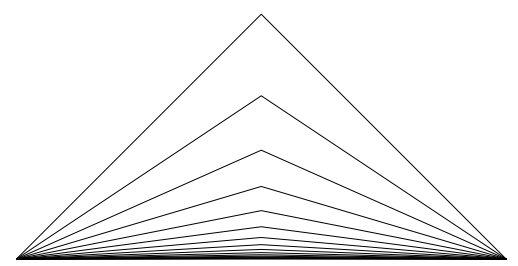

Figure 2. The suspension over a convergent sequence. This continuum is aposyndetic, but not locally connected.

case, it will happen that $Y$ is locally connected, and studying $Y$ will help us gain insight about $J(R)$.

Definition 5 (Aposyndesis). A continuum $X$ is aposyndetic at a point $p \in X$ if every point $q \in X \backslash\{p\}$ is contained in the interior (relative to $X$ ) of a continuum $Y \subset X \backslash\{p\}$.

Aposyndesis is a topological property which is slightly weaker than local connectivity. It is not difficult to see that any locally connected continuum is aposyndetic. Conversely, any aposyndetic continuum which is the boundary of a simply connected domain in $\widehat{\mathbb{C}}$ is locally connected Why39, Theorem 14]. Since the Julia set of a polynomial is the boundary of the domain of attraction of $\infty$, an aposyndetic polynomial Julia set is also locally connected. A simple example of a non-locally connected aposyndetic continuum is illustrated in Figure2. 2 .

Associated to the concept of aposyndesis is the set-valued function $T$, defined below. It should be considered in analogy with a topological closure operator, except that it is not necessarily idempotent.

Definition 6 (The set-valued function $T$ ). For a non-empty set $A \subset X$, let $T(A)$ be the set of $x \in X$ for which all subcontinua of $X \backslash A$ containing $x$ have empty interior relative to $X$. A set $A$ is called $T$-closed if $T(A)=A$.

Remark 7. It follows immediately from the definition that, for any sets $A \subset B$, $A \subset T(A) \subset T(B)$. If $X$ is the $\sin \frac{1}{x}$ continuum depicted in Figure 1 and $A$ is a subset of the limit bar, that $T(A)$ equals the entire limit bar. Further, because every proper subcontinuum of an indecomposable continuum is nowhere dense, $T(A)=X$ whenever $X$ is an indecomposable continuum and $A$ is a subset.

Vought showed that the set-valued function $T$ can be used to define a continuous monotone map from any finished continuum $X$ onto a finite tree $Y$. He does so giving a partition $\mathcal{D}(X)$ of $X$ into subcontinua. The space $Y$ is defined as the quotient space $X / \mathcal{D}(X)$. In general, $m$ is monotone when each element of $\mathcal{D}(X)$ is connected, and $m$ is continuous when the collection $\mathcal{D}(X)$ is upper semicontinuous. The reader is referred to [Nad92, Chapter 3] for these and other facts about decompositions of continua and continuous maps.

The following theorem describes the map defined by Vought.

Theorem 8 (Theorem 1 of Vou74). Let $M$ be a finished continuum which is irreducible about $n$ points, but no fewer. Let

$$
\mathcal{D}(M)=\left\{T^{n}\{x\}: x \in M\right\}
$$

where $T^{n}$ denotes the $n$-fold composition of $T$. Then the following hold: 
(1) $\mathcal{D}(M)$ is an upper semi-continuous decomposition of $M$,

(2) the elements of $\mathcal{D}(M)$ are continua,

(3) the quotient space $M / \mathcal{D}(M)$ is locally connected, and

(4) each element of $\mathcal{D}(M)$ has no interior in $M$.

Further, $\mathcal{D}(M)$ is the only partition satisfying these properties. Also, the quotient $M / \mathcal{D}(M)$ is a tree with $n$ endpoints.

Remark 9. This can be translated as the existence of a continuous monotone map $m: M \rightarrow Y$ where $Y$ is a finite tree with $n$ endpoints and point inverses are nowhere dense in $M$.

For the remainder of the paper, when $X$ is a finished continuum the symbol $\mathcal{D}(X)$ will denote the decomposition provided by this theorem. For a point $x \in X$, let $D(x)$ be the element of $\mathcal{D}(X)$ containing $x$. The following observation relates the properties of $T$-closed sets to the decomposition $\mathcal{D}(X)$.

Lemma 10. Let $X$ be a finished continuum irreducible about $n$ points. Then every $T$-closed set is a union of elements of $\mathcal{D}(X)$.

Proof. Suppose that $A \subset X$ is $T$-closed and let $x \in A$. By Remark $7 x \in$ $T^{n}(\{x\}) \subset T^{n}(A)$, which equals $A$ since $A$ is $T$-closed. But $T^{n}(\{x\}) \in \mathcal{D}(X)$ and $x \in A$ was arbitrary, so $A=\bigcup_{x \in A} D(x)$.

\section{The Decomposition and the Dynamics}

The goal of this section is to prove the following theorem.

Theorem 11. Suppose that $R$ is a rational function whose Julia set is a finitely irreducible continuum.

(1) $J(R)$ contains an indecomposable subcontinuum with non-empty interior relative to $J(R)$, or

(2) $J(R)$ is irreducible between two points, and admits a monotone map $m$ : $J(R) \rightarrow[0,1]$ such that

(a) $m^{-1}(x)$ is nowhere dense for all $x \in[0,1]$, and

(b) $m$ semiconjugates $\left.R\right|_{J(R)}$ to an open, topologically exact, at most $\operatorname{deg}(R)$ to-one map $g:[0,1] \rightarrow[0,1]$.

The main work is to show that the decomposition $\mathcal{D}(J(R))$, defined in the previous section, respects the dynamics of $R$ when $J(R)$ is a finished continuum. Specifically, we aim to show that the image under $R$ of any element of $\mathcal{D}(J(R))$ is again an element of $\mathcal{D}(J(R))$.

First we prove two lemmas relating $T$ and $R$.

Lemma 12. Let $R$ be a rational map. If $A$ is $T$-closed in $J(R)$, then $R^{-1}(A)$ is as well.

Proof. Let $A \subset Y$ be a $T$-closed set, and let $A^{-1}=R^{-1}(A)$. Suppose $x \notin A^{-1}$; we will show that $x \notin T\left(A^{-1}\right)$.

Note that $R(x) \notin A$ since $x \notin T\left(A^{-1}\right) \supset A^{-1}$. Since $A$ is $T$-closed, we have that $R(x) \notin T(A)$. Let $H \subset Y \backslash A$ be a continuum containing $R(x)$ in its interior. Then $R^{-1}(H)$ is a closed set that is also a neighborhood of $x$. Let $H^{-1}$ be the component of $R^{-1}(H)$ containing $x$. Note that $R^{-1}(H)$ is the union of finitely many continua, 
so $H^{-1}$ is open in $R^{-1}(H)$, therefore containing a neighborhood (in $J(R)$ ) of $x$. Also,

$$
\begin{aligned}
H^{-1} \cap A^{-1} & \subset R^{-1}(H) \cap R^{-1}(A) \\
& =R^{-1}(H \cap A) \\
& =\emptyset .
\end{aligned}
$$

Thus $x \notin T\left(A^{-1}\right)$. Since $x$ was any point in $X \backslash A^{-1}$, we see that $T\left(A^{-1}\right)=A^{-1}$.

Theorem 13. Let $R$ be a rational function. If the Julia set $J$ is a finished continuum, then for each $D \in \mathcal{D}(J(R))$ we have $R(D) \in \mathcal{D}(J(R))$.

Proof. Let $Y=J(R) / \mathcal{D}(J(R))$, and let $m: J(R) \rightarrow Y$ be the associated quotient map. Recall from Theorem 8 that elements of $\mathcal{D}(J(R))$ are nowhere dense in $J(R)$ and that $Y$ is a finite tree. For $x \in J(R)$, recall that $D(x)$ denotes the unique element of $\mathcal{D}(J(R))$ containing $x$. We must show that $R(D(x))=D(R(x))$. Let $K$ be the component of $R^{-1}(D(R(x)))$ containing $x$. Because $R$ is confluent, we see that $R(K)=D(R(x))$. It is then sufficient to show that $D(x)=K$.

We see that $K$ has empty interior in $J(R)$, since $R(K)=D(R(x))$ has empty interior in $J(R)$ and $\left.R\right|_{J(R)}$ is an open map. As a component of a $T$-closed set, $K$ is $T$-closed [FS67, Lemma 2.6] and the union of elements of $\mathcal{D}(J(R))$ by Lemma 10 . Therefore, $K=m^{-1}(m(K))$, so $m(K)$ must have empty interior in $Y$. However, $Y$ is a finite tree, so the only subcontinua with empty interior are points. We then conclude that $m(K)$ is a point, and therefore $K$ is an element of $\mathcal{D}(J(R))$.

Now we study the induced map $g: J(R) / \mathcal{D}(J(R)) \rightarrow J(R) / \mathcal{D}(J(R))$ and use it to draw conclusions about the set $J(R)$ itself.

Theorem 14. Suppose that $R$ is a rational map whose Julia set is a finished continuum. Then the monotone map $m: J(R) \rightarrow J(R) / \mathcal{D}(J(R))$ is a monotone semiconjugacy of $\left.R\right|_{J(R)}$ to a map $g: J(R) / \mathcal{D}(J(R)) \rightarrow J(R) / \mathcal{D}(J(R))$ of a finite tree. The map $g$ is then open, topologically exact, and at most $\operatorname{deg}(R)$-to-one.

Proof. For brevity, set $Y=J(R) / \mathcal{D}(J(R))$. Notice that $m \circ R$ is constant on every set of the form $m^{-1}(y)$ by Theorem 13. Therefore, the map $g: Y \rightarrow Y$ defined by $g=m \circ R \circ m^{-1}$ is a single-valued, continuous map (see [Mun75, Theorem 22.2]).

To see that $g$ is open and topologically exact, let $U \subset Y$ be open. Then $m^{-1}(U) \subset J(R)$ is an open set, saturated with respect to the quotient map $m$. Since $\left.R\right|_{J(R)}$ is open, $R\left(m^{-1}(U)\right) \subset J(R)$ is open, and by Theorem 13 this set is also saturated. Therefore, $m\left(R\left(m^{-1}(U)\right)\right)=g(U) \subset Y$ is open, so $g$ is an open map. Also, since $m^{-1}(U) \subset J(R)$ is open, there exists $n \in \mathbb{N}$ such that $R^{n}\left(m^{-1}(U)\right)=J(R)$. Thus $m \circ R^{n} \circ m^{-1}(U)=g^{n}(U)=Y$, so $g$ is topologically exact.

To show that $g$ is at most $\operatorname{deg}(R)$-to-one, let $y \in Y$. Then $R^{-1}\left(m^{-1}(y)\right)$ is the union of at most $\operatorname{deg}(R)$ elements of $\mathcal{D}(J(R))$, since each element must map onto $m^{-1}(y)$ and hence must contain a preimage of $y$. Therefore, $m\left(R^{-1}\left(m^{-1}(y)\right)\right)=$ $g^{-1}(y)$ has cardinality at $\operatorname{most} \operatorname{deg}(R)$.

Corollary 15. If $R$ is a rational function and $J(R)$ is a finished continuum, then $J(R) / \mathcal{D}(J(R))$ is an arc, and $J(R)$ is irreducible. 
Proof. Suppose that $J(R) / \mathcal{D}(J(R))$ has a cut point $x$ of order at least 3. Since $g$ is topologically exact, we have that $B=\bigcup_{n \in \mathbb{N}} g^{-n}(x)$ is dense in $J(R) / \mathcal{D}(J(R))$. Since $g$ is open, each point of $B$ is also a branch point of $J(R) / \mathcal{D}(J(R))$. Therefore, $J(R) / \mathcal{D}(J(R))$ has infinitely branch points if it has one. A finite tree does not have infinitely many branch points, so $J(R) / \mathcal{D}(J(R))$ is an arc. That $J(R)$ is irreducible follows from Theorem 8 and that the arc is an irreducible continuum.

Remark 16. It is known that topologically exact open maps of intervals are conjugate to $n$-saw maps, which are of the form

$$
f(x)= \begin{cases}(n-2 i) x & \text { if } x \in[2 i, 2 i+1] \\ (2(i+1)-n) x & \text { if } x \in[2 i+1,2 i+2] .\end{cases}
$$

Therefore, $g$ is conjugate to a map of this sort.

Combining the above, we can prove the main theorem of the section.

Proof of Theorem 11. Suppose that $J$ contains no indecomposable subcontinuum with interior. The monotone map $m$ is the quotient map corresponding to the decomposition of Theorem 8 . That $m$ is an arc is Corollary [15, and the facts about the induced map $g: I \rightarrow I$ are from Corollary 14.

\section{The Polynomial Case}

In the case of polynomial Julia sets, we can say more.

Theorem 17. Let $P$ be a polynomial, and suppose that $J(P)$ is finitely irreducible. Then either

(1) $J$ is an indecomposable continuum, or

(2) $J$ is homeomorphic to an arc.

Proof. Suppose that $J$ is not indecomposable. Then no indecomposable subcontinuum has interior in $J$ CMR06, Theorem 1], so $J(P)$ is a finished continuum. Let $m: J \rightarrow[0,1]$ be the monotone map provided by Theorem [11, and let $g: I \rightarrow I$ be the map to which $\left.P\right|_{J(P)}$ is conjugate via $m$. We will show that $J(P)$ is homeomorphic to an arc by showing it is locally connected (see Remark 2). This will be accomplished by showing that the forward orbits of its critical points are finite Mil06, Theorem 19.7].

For $t \in[0,1]$, let $J_{t}$ denote the union of the continuum $m^{-1}(t)$ with its bounded complementary domains. It is not difficult to see that $P\left(J_{t}\right)=J_{g(t)}$, and that the $\left.\operatorname{map} P\right|_{J(t)}: J_{t} \rightarrow J_{g(t)}$ is open for each $t \in I$ (for instance by [Nad92, Lemma 13.13]).

Extend $m: J(P) \rightarrow[0,1]$ to a map $\hat{m}: \bigcup_{t \in[0,1]} J_{t} \rightarrow[0,1]$ by sending points of $J_{t}$ to the point $t \in[0,1]$. Each $\hat{m}^{-1}(t)$ is a non-separating plane continuum, so the Vietoris-Begle theorem implies that $\hat{m}$ induces an isomorphism between the Cech cohomologies of $\bigcup_{t \in[0,1]} J_{t}$ and $[0,1]$. Therefore, $\bigcup_{t \in[0,1]} J_{t}$ is a non-separating plane continuum containing $J(P)$, so $\bigcup_{t \in[0,1]} J_{t}$ equals the filled Julia set. Since $J(P)$ is connected, each critical point is contained in the filled Julia set [Mil06, 9.5] and therefore in some $J_{t}$.

We now show that a point $t \in[0,1]$ is a critical point of $g$ if and only if $J_{t}$ contains a critical point of $P$. Suppose that a fiber $J_{t}$ does not contain any critical point of $P$. Then there is a (saturated) neighborhood $U$ of $J_{t}$ on which $P$ is a 
homeomorphism. In particular, no two fibers contained in $U$ have the same image, so $g$ is a homeomorphism on $m(U)$.

On the other hand, suppose that $t$ is not a critical point of $g$. Then there is a (saturated) neighborhood $U$ of $J_{t}$ such that no two fibers contained in $U$ have the same image. If any fiber $J_{t_{0}}$ does not map by a homeomorphism onto its image, then $J_{t_{0}}$ must contain a critical point since it and its image are full continua. Let $V \subset U$ be a disk about $J_{t}$ such that $\left.R\right|_{V \backslash J_{t}}$ is a covering map onto its image. Then a fiber which does not contain a critical point intersects $V$, and $R$ is one-to-one in a neighborhood of it. Therefore, $\left.R\right|_{V}$ is a homeomorphism, and $J_{t}$ cannot contain a critical point.

If one takes a saturated open set $V \subset U$ about $J_{t}$ such that $V \backslash J_{t_{0}}$ does not contain the preimage of any critical value, we see that $\left.R\right|_{V}$ is a homeomorphism, so $J_{t}$ contains no critical point of $R$.

Notice that $J_{0} \cup J_{1}$ is a forward-invariant set. Suppose without loss of generality that $P\left(J_{0}\right)=J_{0}$. Note that $J_{0}$ cannot contain a critical point of $P$, since 0 cannot be a critical point of $g$. Therefore, $\left.P\right|_{J_{0}}$ is a forward expanding homeomorphism, which implies that $J_{0}$ must be a point [Mil06, Lemma 18.8]. Then $J_{1}$ must also be a point, either because it is fixed or because it maps to $J_{0}$. Because every critical point of $g$ must map into $\{0,1\}$, every critical point of $R$ must map into $J_{0} \cup J_{1}$ and hence has a finite orbit. This implies that $J(P)$ is locally connected, and therefore an arc.

\section{FURTHER WORK}

The main novelty in this work is the use of what might be called a locally connected model of the Julia set to draw conclusions about its topology and the dynamics of its rational map. There is more structure on polynomial Julia sets than there are on rational Julia sets, so it is to be expected that more can be concluded in the polynomial case. Locally connected models for polynomial Julia sets have been studied further in joint work with Alexander Blokh and Lex Oversteegen in BOC08, where we characterized the finest locally connected model for the action of a polynomial on its Julia set.

Vought's decomposition is rather special, and only applies to finitely irreducible continua. It is an example of a broader notion called a core decomposition. A decomposition is core with respect to a property $\mathrm{P}$ if it has property $\mathrm{P}$ and it refines all decompositions with property P. Therefore, Vought's decomposition for finished continua is core with respect to the property that the quotient space is locally connected.

Generally speaking, there is no core decomposition of an arbitrary continuum with locally connected quotient. However, there is always a core decomposition with an aposyndetic quotient FS67, of which Vought's decomposition is a special case. Such a model may serve in the stead of a locally connected model where locally connected models are unavailable. However, the utility is limited when the

continuum in question contains an indecomposable subcontinuum with interior, as it must be absorbed into an element of any decomposition to a nice space. There are several pertinent questions.

Question 18. Does there exist a rational function whose Julia set contains an indecomposable continuum with interior? 
Question 19. Does there exist a rational function whose Julia set does not have a finest locally connected model?

Question 20. Let $R$ be a rational function with connected Julia set. Is the finest decomposition to an aposyndetic continuum invariant with respect to $R$ ?

Question 21. For what useful topological properties $P$ does there exist a finest decomposition of every Julia set $J(R)$ satisfying $P$ ? Is the decomposition dynamic? Which of these is the appropriate analog for the finest locally connected model?

\section{REFERENCES}

[BOC08] Alexander M. Blokh, Lex G. Oversteegen, and Clinton P. Curry. Locally connected models for Julia sets. Preprint, 2008. arXiv:0809.3754v1 [math.DS]., 2008.

[CMMR] Clinton P. Curry, John C. Mayer, Jonathan Meddaugh, and James T. Rogers, Jr. Any counterexample to Makienko's conjecture is an indecomposable continuum. Accepted to Ergodic Theory and Dynamical Systems. arXiv:0805.3323 1 [math.DS].

[CMR06] Douglas K. Childers, John C. Mayer, and James T. Rogers, Jr. Indecomposable continua and the Julia sets of polynomials. II. Topology Appl., 153(10):1593-1602, 2006.

[FS67] R. W. FitzGerald and P. M. Swingle. Core decomposition of continua. Fund. Math., 61:33-50, 1967.

[Jon41] F. Burton Jones. Aposyndetic continua and certain boundary problems. Amer. J. Math., 63:545-553, 1941.

[Jon52] F. Burton Jones. Concerning aposyndetic and non-aposyndetic continua. Bull. Amer. Math. Soc., 58:137-151, 1952.

[Mil06] John Willard Milnor. Dynamics in one complex variable, volume no. 160. Princeton University Press, Princeton, 3rd ed edition, 2006.

[Mun75] James R. Munkres. Topology: a first course. Prentice-Hall Inc., Englewood Cliffs, N.J., 1975.

[Nad92] Sam B. Nadler, Jr. Continuum theory. An introduction., volume 158 of Monographs and Textbooks in Pure and Applied Mathematics. Marcel Dekker Inc., New York, 1992.

[Vou74] Eldon J. Vought. Monotone decompositions into trees of Hausdorff continua irreducible about a finite subset. Pacific J. Math., 54(2):253-261, 1974.

[Why39] G. T. Whyburn. Semi-locally connected sets. Amer. J. Math., 61:733-749, 1939.

(Clinton P. Curry) Department of Mathematics, University of Alabama at Birmingham, Birmingham, AL 35294-1170

E-mail address: clintonc@uab.edu

URL: http://www.math.uab.edu/ curry 